One cat had a soft tissue sarcoma arising at the level of the nasal planum.

In all cases, apart from one Sr90 was used as adjuvant treatment to surgery. Different types of protocol were used. Seven of the ten cases received a protocol involving five fractions of 30-40 Gy each, administered on alternating days; three cases received $\mathrm{Sr} 90$ as a single fraction of 80 Gy. In two cases Sr90 plesiotherapy was repeated after tumour recurrence. The treatment was overall very well tolerated with minimal side effects.
The overall outcome was good. Two cases experienced recurrence of the neoplasia in the radiation field or adjacent areas (two cats: one sarcoid and one progressive dendritic histiocytosis). The rest of the cases showed no evidence of recurrence of the disease on a follow-up period of 10 to 839 days.

In conclusion, this study showed that Sr90 plesiotherapy is a valid and well-tolerated alternative for the control of local disease for different types of tumours arising in difficult areas, where other treatment options (e.g. external beam radiotherapy) may not be feasible.

\section{Assessment of an in vitro lymphocyte response to chemotherapy agents as a guide to treatment in canine lymphoma}

\section{Chiara Giannasi', Simon Tappin', Rob Foale', Kevin Slater ${ }^{2}$, Ilias Alexandrakis ${ }^{2}$}

1 Dick White Referrals, Station Farm, London Road, Six Mile Bottom, Cambridgeshire, UK

2 Avacta Animal Health, Unit 706, Avenue E, Thorp Arch Estate, Wetherby, UK

\section{INTRODUCTION}

The ability to identify a tumour's potential sensitivity to chemotherapy pre-treatment, or at relapse, might guide selection of chemotherapy protocol and thereby improve outcome. In the human field, assessment of the in vitro response of individual patient's tumour to chemotherapy has proved controversial. This study aimed to develop an assay for canine lymphoma, assessing pre-treatment in vitro lymphocyte responses to chemotherapy drugs and to correlate this with clinical outcome. In addition, we aimed to try to identify evidence of chemotherapy resistance at the point of relapse.

\section{METHODS}

Eleven dogs with multi-centric Iymphoma were included in this study with informed consent; all were treated with a standardised $\mathrm{CHOP}$ protocol. The average age of the dogs included was 93 months, with 6 males (2 entire, 4 neutered) and 5 females ( 2 entire, 3 neutered). Eight dogs had T-cell, and three had B-cell lymphoma (grade III $(n=6)$, IV $(n=2)$ and $V(n=3)$, sub-stage $a(n=5)$ and $b(n=6))$. Fine needle aspirates of the most prominent peripheral lymph nodes were collected pre-treatment and cultured in 96 well microplates in serum free DMEM under conditions previously shown to favour growth of malignant cells. In an attempt to predict cells' in vivo sensitivity to chemotherapy, vincristine, cyclophosphamide, L-asparaginase and doxorubicin were added to test wells at concentrations equivalent to each drugs reported serum cMax. After five days culture, cell viability was measured using the ATP bioluminescence technique. Percentage inhibition occurring in response to the drugs was calculated with reference to drug-free control wells. Correlation between percentage inhibition, time to relapse and subsequently overall survival time was calculated.

\section{RESULTS}

The results showed no correlation between cell inhibition and both, time to relapse and overall survival. There was no difference in the predictive value of response to treatment in naïve dogs compared with dogs that had received several doses of chemotherapy. Repeat samples were taken from two dogs at relapse (at weeks 18 and 39) and there was no clear evidence of chemotherapy resistance in either dog.

\section{CONCLUSION}

This in vitro assay in its current form appears to add no additional information on an individual's response to chemotherapy. Further study, including analysis of longer culture periods and the effect of different drug concentrations on cell growth, is needed to better determine and correlate in vitro response to chemotherapy.

\section{Evaluation of lomustine for the treatment of canine T-cell lymphoma}

\section{Chiara Giannasi, Simon Tappin, Rob Foale}

Dick White Referrals, Station Farm, London Road, Six Mile Bottom, Cambridgeshire, UK

\section{INTRODUCTION}

The current first line standard of care treatment for canine T-cell lymphoma is a multidrug doxorubicin-based chemotherapy protocol (eg: CHOP). Use of single agent lomustine has been described as a rescue therapy. Recently, lomustine has been proposed as an alternative first line treatment. The aim of this study was to assess the efficacy of lomustine in a referral population of dogs with T-cell lymphoma as a first line and rescue therapy. 


\section{Oral presentations}

\section{METHODS}

Medical records of 16 dogs treated with lomustine for multicentric T-cell lymphoma were retrospectively reviewed. Ten dogs were treated with a CHOP based protocol (three of which had the inclusion of L-asparaginase initially) and received lomustine as a rescue agent once remission was lost. Six dogs were treated with lomustine as first line therapy for T-cell lymphoma. All dogs received single agent lomustine with a median starting dose of $78.5 \mathrm{mg} / \mathrm{m}^{2}$ (range $60-90 \mathrm{mg} / \mathrm{m}^{2}$ ) every three weeks. Dose adjustment and increased dosage intervals were used when myelosuppression was documented. A total of eight treatments were planned in all dogs. Additional prednisolone was administered in three dogs (starting dose $0.5-1.7 \mathrm{mg} / \mathrm{kg}$ q24hrs tapered over four weeks). For those completing therapy, drug-free remission periods were evident. Response to treatment, time to relapse, and survival times for all dogs were documented. Signs of relapse included clinical deterioration and recurrence of lymphadenomegaly and cytological confirmation of lymphoma from aspirates obtained from enlarged lymph nodes where possible.

\section{RESULTS}

Of the dogs treated with lomustine as a first line agent $(n=6)$ four dogs had died as a result of their disease with a median time to relapse of 57.5 days (range: 44-105). Two dogs were still alive 628 and 1095 days after starting therapy. The median time to relapse for the dogs treated with $\mathrm{CHOP}$ prior to receiving lomustine was 112 days (range: 49-322). Eight of these dogs had a response to lomustine as rescue therapy. Three dogs achieved complete remission, five had a partial response or stable disease and two had no response to therapy with progressive lymph node enlargement. Dogs that responded to lomustine as a rescue agent had a median remission period of 50 days (range: 18-649).

\section{CONCLUSION}

Lomustine used first line appears to offer a good long term outcome to a proportion of dogs with T-cell lymphoma, further work is required to investigate this finding.

\section{Spatial clustering of neoplasia in a population of dogs from UK first opinion practice}

\section{Stephanie Marlow, Anneliese Stell, Kate English, David Brodbelt}

Royal Veterinary College, London, UK

\section{INTRODUCTION}

There is limited research into the existence of spatial patterns of neoplasia in the UK general canine population. In addition, until now, there have been few sources of veterinary clinical data that reflect the general population of dogs in the UK and that would therefore enable such investigation. This study aimed to analyse spatial patterns of neoplastic disease in general and mast cell tumours (MCTs) specifically, in the UK first opinion practice-attending canine population, in order to generate hypotheses regarding environmental risk factors for neoplasia.

\section{METHODOLOGY}

All data derived from the database of the 'VetCompass' programme (Veterinary Companion Animal Surveillance System); which collects de-identified first opinion practice data directly from electronic patient records from over 450 recruited clinics. A case control study was undertaken to evaluate spatial clustering of neoplasia in general and MCTs. Cases were defined as dogs attending one of the VetCompass clinics included in this study, with a neoplasm confirmed via histopathology, or cytology reported by a specialist centre (2010-12). MCTs were the most frequently diagnosed potentially malignant neoplasm and were thus taken forward for individual spatial analysis. Partial postcodes for all cases and controls were extracted from the database and their centroid coordinates established via an online Doogle tool using the mean longitude and latitude of all potential postcodes corresponding to the area of the partial postcode. All mapping was completed in QGIS 2.6.1. Presence of clusters with statistically significant high/low relative risk, were identified via the spatial scan statistic using SaTScan software and a Bernoulli probability model. Statistical significance was set at $5 \%$.

\section{RESULTS}

There was evidence of statistically significant clustering for the presence of neoplasia overall and also among the MCTs. An increase in relative risk in and around London was apparent for neoplasia overall with a reduced risk in Cambridgeshire and Oxfordshire. Interestingly, one of those areas of high relative risk identified for neoplasia overall centred in Edgware, Greater London, was in contrast an area of low relative risk for MCTs, while a cluster of high relative risk for MCTs was centred in Hertfordshire.

\section{CONCLUSION}

Clustering of canine tumours may reflect differential geographical risk of neoplasia. These data, though preliminary, provide a useful base for more detailed analysis of geographical risk factors for neoplasia within the UK general canine population. 